\title{
Forces between colloidal droplets in the presence of a weak polyelectrolyte
}

\author{
JOHN PHILIP*, O MONDAIN MONVAL ${ }^{\dagger}$, F LEAL-CALDERON ${ }^{\dagger}$ and J BIBETTE ${ }^{\dagger}$ \\ Metallurgy and Materials Group, Indira Gandhi Centre for Atomic Research, Kalpakkam 603 102, India \\ ${ }^{\dagger}$ CRPP-CNRS, Avenue Albert Schewitzer, 33600 Pessac, France
}

\begin{abstract}
In this paper, we present the results on the forces between individual colloidal liquid droplets in the presence of a weak polyelectrolyte, poly(acrylic acid), using magnetic chaining technique. The effect of the repulsive forces have been investigated under different experimental conditions such as polyelectrolyte concentration, adsorption time, salt concentration etc. At a PAA concentration of 0.01\% (weight), a long range repulsive force profile is observed due to the adsorption of polyelectrolyte on the droplet, without any irreversible aggregates even at very small inter-droplet spacing. Above a concentration of 0.01 wt $\%$ of PAA, formation of irreversible chaining of droplets is observed at short inter-droplet separations due to polyelectrolyte bridging. The onset of binding is also independently confirmed by microscopic observation. Compared to the slow adsorption on mica surfaces, the PAA adsorption on the colloidal droplets is found to be rapid. Up to $0.1 \mathrm{M} \mathrm{NaCl}$, the range of repulsion and the hydrodynamic radius of the droplet is found to be increasing.
\end{abstract}

Keywords. Colloidal droplets; polyelectrolyte.

\section{Introduction}

Colloid is a generic name for any dispersion in which the characteristic size of the dispersed domains is between $10 \mathrm{~nm}$ and $100 \mathrm{~m}$. This includes numerous systems such as foams (a gas dispersed in a liquid), smoke (a solid dispersion in a gas), emulsions (one liquid dispersed in another) or paints (a solid dispersed in a liquid). Applications of colloidal science are in constant progress in many aspects of life. Colloids are used in a huge number of processes such as extracting oil from geological deposits, aerosols for dispensing domestic products like shaving creams and deodorants, sprays containing weedicide and insecticides, emulsions, gels, food processing, packaging industry etc.

Colloids generally require repulsive surface forces to become meta stable. The most important forces which determine the stability of colloidal dispersions are van der Waals dispersion forces, electrostatic and steric forces due to polymers adsorbed at the interface. Other forces that are important at short intermolecular distances are solvation and hydrophobic forces. The classical Derjaguin-Landau-Verwey-Overbeck (DLVO) theory combined the van der Waals forces with the electrostatic double-layer forces to explain the stability of lyophobic colloids. The net force acting between the colloidal particles determines the stability of the colloidal system. A net attractive force leads to the aggregation or clustering

*Author for correspondence of particles or for liquid particles coalescence may occur and the system will be unstable. If the net force is repulsive, the system will remain stable. The height of the potential barrier decides the stability of the colloidal system. Force measurement techniques have been widely used to understand the interactions that give insight into the colloidal stability (Israelachvili 1985).

Most of the time, electrostatically stabilized colloids become unstable when the ionic strength of the medium is increased sufficiently, due to the reduction in the spatial extension of electrical double layers (e.g. clays, sols, lattices). Therefore, one major advantage of using macromolecules as stabilizers is that their double layers are less sensitive to electrolyte concentration. As a result, steric stabilization of colloidal dispersions has triggered a lot of interest in recent years due to its advantages over electrostatic counterpart and more importantly due to its numerous industrial applications (Napper 1983; Patel and Tirrell 1989).

Stabilization of the colloidal dispersions with macromolecules has been explored in various technological applications for many years, however, the interactions between polymer bearing surfaces could be studied directly only after the introduction of the surface force measurement apparatus (Israelachvili and Tabor 1972; Israelachvili and Adams 1978; Luckham and Klien 1984). The elucidation of the structure of the adsorbed layer on the colloidal emulsion is important since it plays a major role in the stabilization of the colloids. Steric stabilization of the colloidal dispersions has triggered a 
lot of interest due to its several advantages over its electrostatic counterpart and more importantly due to its numerous industrial applications (Sato and Ruchs 1980; Napper 1983; Th Tadors 1983, 1988). It is well known that electrostatically stabilized colloids often coagulate when the ionic strength of the medium is increased sufficiently, due to the reduction in the spatial extension of the electrical double layers. Ionic strength higher than $0.001 \mathrm{M}$, corresponds to a length of about $10 \mathrm{~nm}$ for a $1: 1$ electrolyte and can lead to van der Waals attraction in the dispersion. This is the range where electrostatic stabilization often fails. One major advantage of using macromolecules as stabilizers is that the double layers are less sensitive to electrolyte concentration.

Measurement of forces acting between solid surfaces has been a topic of intense research over the last two decades (Israelachvili 1985). Most of the force measurements have been carried out by using the novel surface force apparatus (SFA) introduced by Israelachvili and Tabor (1972). Other relatively new force measuring techniques (Hansma et al 1988; Prieve and Frej 1990) such as total internal reflection microscopy, atomic force microscopy etc have also been used for such investigations but occasionally compared to SFA. Recently, a new technique (Leal et al 1994), called magnetic field induced chaining technique (MCT) has been introduced which allows the measurement of forces between two individual emulsion droplets. In contrast to other older techniques, this method leads to the direct in situ measurement at the colloidal scale. In SFA, the force is measured between the semi macroscopic surfaces (mica) whereas in MCT the forces between individual colloidal droplets are measured. Using this technique, one can measure the colloidal forces in a wide variety of materials encountered in emulsions and dispersions. The present work is mainly aimed to understand the stability of emulsion droplets in the presence of polyelectrolyte. We present the force profiles-distance profiles in the absence and presence of polyelectrolytes (Philip 1995).

\section{Materials}

\subsection{Ferrofluid}

Ferrofluid oil consisted of a collection of ferromagnetic domains of $\mathrm{Fe}_{2} \mathrm{O}_{3}$ dispersed in the octane and was supplied by Rhone Poulenc Inc. The typical size of the $\mathrm{Fe}_{2} \mathrm{O}_{3}$ particles was about $10 \mathrm{~nm}$. The inner surfactant used to stabilize the oxide particles against van der Waals attraction was oleic acid. The polar group of the adsorbed oleic acid associated with the oxide particle surface and disguised the surface to look like part of the continuous phase (octane). The technique used to make the stock emulsion was the classical inversion method (Becher 1965). The fractionation technique
(Bibette 1991) was used to select emulsion droplets with a diameter of about $200 \mathrm{~nm}$. The size distribution of the final emulsion was measured by using a Malvern Instruments Master sizer (Ver. 2.14).

\subsection{Polyelectrolyte}

Poly(acrylic acid) (PAA) of molecular weight of 250,000 $\left(M_{\mathrm{w}} / M_{\mathrm{N}}=1 \cdot 10\right)$ was supplied by Rhone-Poulenc and Aldrich Chemicals. PAA is a weak acid and its monomer is shown below

$$
\left[-\mathrm{CH}_{2}-\mathrm{CH}\left(\mathrm{CO}_{2} \mathrm{H}\right)\right] \text {. }
$$

The unperturbed radius of gyration of this polymer in good solvent (water) was found to be $35 \mathrm{~nm}$ (Brandrupp and Immergutt 1975). PAA is a water soluble polyelectrolyte and it can exhibit a large variety of behaviours due to the amphiphilic nature of its constituents. Hydrophobic nature of the backbone makes the solubilization of the uncharged chains difficult. Theta temperature for PAA in water is about $20^{\circ} \mathrm{C}$. The solubilization of PAA in water at room temperature is due to electrostatic repulsion between ionized carboxylic groups along the chain. The non-ionic surfactants used for these investigations was Nonyl phenol Ethoxylate (NP10).

$$
\mathrm{C}_{9} \mathrm{H}_{19}-\mathrm{C}_{6} \mathrm{H}_{4}-\mathrm{O}-\left(\mathrm{CH}_{2} \mathrm{CH}_{2} \mathrm{O}\right)_{10}-\mathrm{H} \text {. }
$$

\section{Experimental}

Ferrofluid oil was used and the droplets were paramagnetic in nature (Rosensweig 1985). An applied field induces a magnetic dipole in each drop, causing them to form chains. Without external field, these droplets have no permanent magnetic moments because of the random orientation of the magnetic grains within the droplets, due to thermal motion. An external magnetic field orients these magnetic grains slightly toward the field direction, which results in a dipole moment in each droplet. The magnitude of the magnetic dipole moment increases with the strength of the applied field until saturation is reached. The strength of this dipolar interaction can be described by a coupling constant, which is the ratio of the contact dipole energy of two parallel dipoles to the thermal energy (Mou et al 1994). At low concentration, one-droplet thick chains are well separated and oriented along the field direction. Due to the presence of the one-dimensional ordered structure, Bragg peak can be observed, from which the inter-droplet separation is estimated precisely. The condition for forming a linear chain is that the repulsive force between the droplets must exactly balance the attractive force between the droplets induced by the applied magnetic field. The spacing between droplets is directly measured from the 
determination of the spectral distribution of the scattered light at a constant angle. For perfectly aligned particles with a separation ' $d$ ', the first order Bragg condition leads to $2 d=\lambda_{0} / n$. Where $n$ is the refractive index of the suspending medium ( $n=1.33$ for water) and $\lambda_{0}$ is the wavelength of the light Bragg scattered at an angle of $180^{\circ}$. The peak position moves toward smaller as the field is increased. Because the droplets are monodispersed and negligibly deformable owing to their large capillary pressure, the corresponding interfacial separation is $h=d-2 a$. The magnitude of the induced dipole is controlled by the strength of the applied field. From the dipolar interaction, the corresponding repulsive force between the droplet interface is calculated (Leal et al 1994; Mondain-Monval et al 1995).

\section{Results and discussion}

\subsection{Force profiles in the presence of an anionic surfactant SDS}

The repulsive force between the colloidal droplets stabilized with an anionic surfactant (SDS) at two concentrations, $8 \times 10^{-3} \mathrm{M}$ and $8 \times 10^{-4} \mathrm{M}$, are shown in figure 1 . Here, the electrostatic repulsion arises from the presence of anionic surfactant (SDS) molecules adsorbed on the colloidal droplet. The estimated values of debye lengths at these concentrations are respectively $3.4 \mathrm{~nm}$ and $10.7 \mathrm{~nm}$. Due to the changes in the surfactant concentration, the corresponding surface potential and debye length changes. For the lower concentration, the counterion layer extends well beyond the surface and become diffused. This results in the long range electrostatic repulsion. At a higher surfactant concentration (CMC of SDS $=8 \times 10^{-3} \mathrm{M}$ ), most of the counter ions are located within a few nanometres from the surface of the droplets that leads to a short range repulsion. This observation

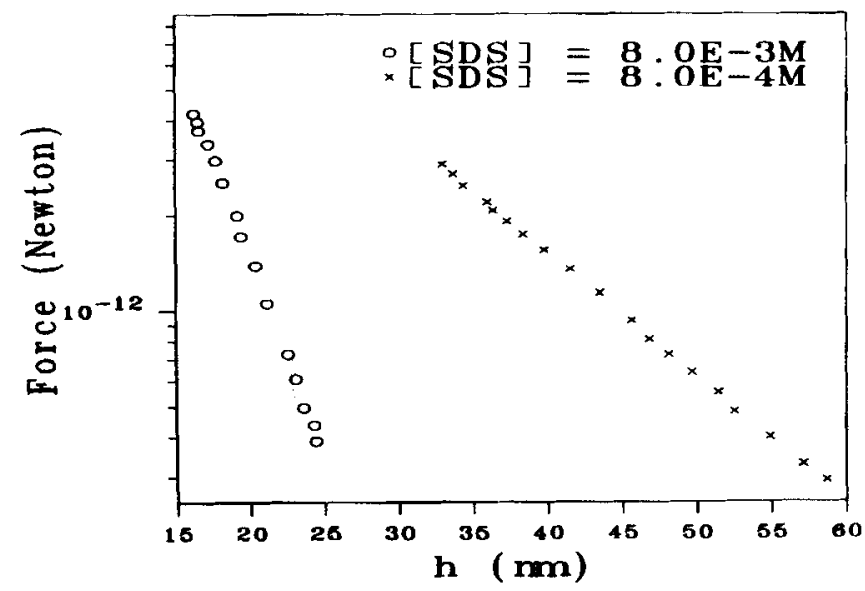

Figure 1. Force distance profiles with ionic surfactant SDS of two different concentrations. shows that the spatial extension of the double layer is very sensitive to the concentration (or ionic strength) of surfactant.

The minimum force shown in the figure 1 corresponds to the force sufficient to hold the particles within a chain, and the corresponding interaction energy is necessarily greater than the thermal energy $k T$. The maximum force is determined by the saturation field of the ferrofluid, which may eventually be increased by an order of magnitude by using a ferrofluid with larger susceptibility. The minimum and maximum force measured using this technique are $10^{-13} \mathrm{~N}$ and $10^{-11} \mathrm{~N}$, respectively and the resolution of interfacial separation is about $1 \mathrm{~nm}$.

For particles having small charge densities, the force between two particles is given by (Verway and Overbeck 1948)

$$
F_{\mathrm{r}}(d)=4 \pi \varepsilon \Psi_{0}^{2} a^{2}\left(\frac{\kappa}{d}+\frac{1}{d^{2}}\right) \exp [-\kappa(d-2 a)],
$$

where $\varepsilon$ is the dielectric permittivity of the suspending medium, $\Psi_{0}$ the electrical surface potential and $\kappa$ the inverse Debye length. The above equation is valid for extended double layers where $\kappa a<5$. If the particles have thin double layer, $\kappa a>5$, the alternate Derjaguin (1940) approximation provides the approximate interaction force with the assumption that $\Psi_{0}$ remains constant independent of $h$;

$$
F_{\mathrm{r}}(d)=2 \pi \varepsilon \Psi_{0}^{2} a \kappa \frac{\exp [-\kappa(d-2 a)]}{1+\exp [-\kappa(d-2 a)]} .
$$

The best fit obtained with the surface potential using the above equation is shown in figure $2(\kappa a>5)$ for a surfactant concentration of $8 \times 10^{-4} \mathrm{M}$. The surface potential obtained from the best fit was $-41 \mathrm{mV}$.

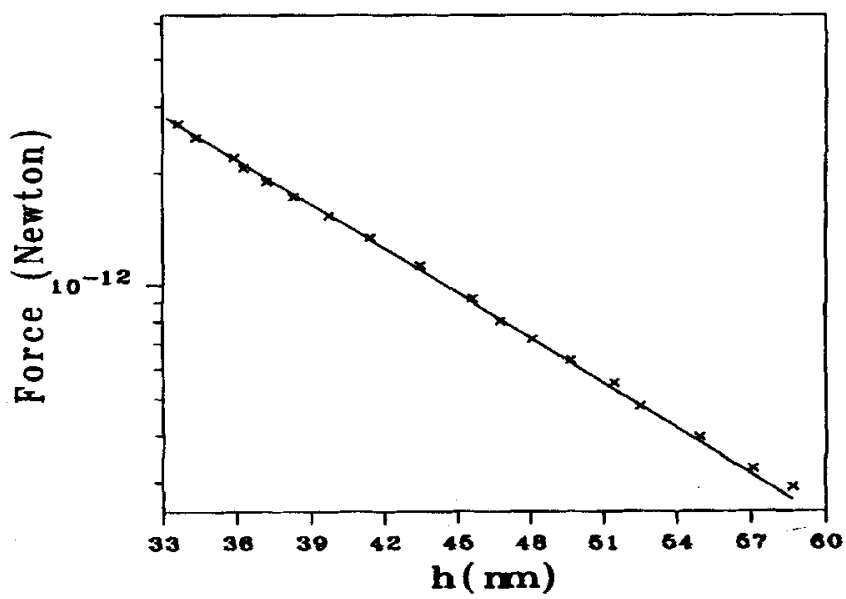

Figure 2. Force distance profile with ionic surfactant SDS and the best fit (continuous line) using (2). 


\subsection{Force profiles in the presence of a non-ionic} surfactant NPIO

The initial emulsion is based on anionic surfactant (SDS) and the droplet surface is negatively charged. In order to remove the charges from the droplet surface, a small quantity of the SDS stabilized ferrofluid emulsion is washed with large amount of nonionic surfactants (NP10) many times. It is easy to check whether the droplet interface is charge free, from the force measurement experiment. When the droplets are charge free, a clean hard sphere profile can be seen at short interdroplet separation. We use such emulsions (which show hard sphere profiles) for our polyelectrolyte studies. So, any variation from this hard sphere profile will be due to the polyelectrolyte in the system.

The force distance profile for the ferrofluid droplets stabilized with a non-ionic surfactant NP10 at two pH values, four and six, are shown in figure 3. Since NP10 is a non-ionic surfactant, one would expect a hard sphere profile when the surfactant is adsorbed on the droplet. In contrary to this, we have observed an electrostatic repulsive force profile at $\mathrm{pH}=6$. When the $\mathrm{pH}$ of the NP10 solution is changed to four, a clean hard sphere profile is observed. At $\mathrm{pH}=4$, the colloidal system was stable for several months whereas $\mathrm{pH}$ below three, the system was unstable. One could also argue the possibility of the iron oxide particles denuded of protective fatty layers partition themselves at the interface and that could lead to double layer repulsion observed at $\mathrm{pH}=6$. We clearly rule out this possibility because of following reasons: The moment the particles denude, the ferrofluid emulsions become unstable and lots of aggregates can be seen under a microscope. Before we start any experiment, we observe the emulsion under a microscope and make sure that the emulsion is stable and aggregates free. If the fluid droplet becomes unstable in the presence

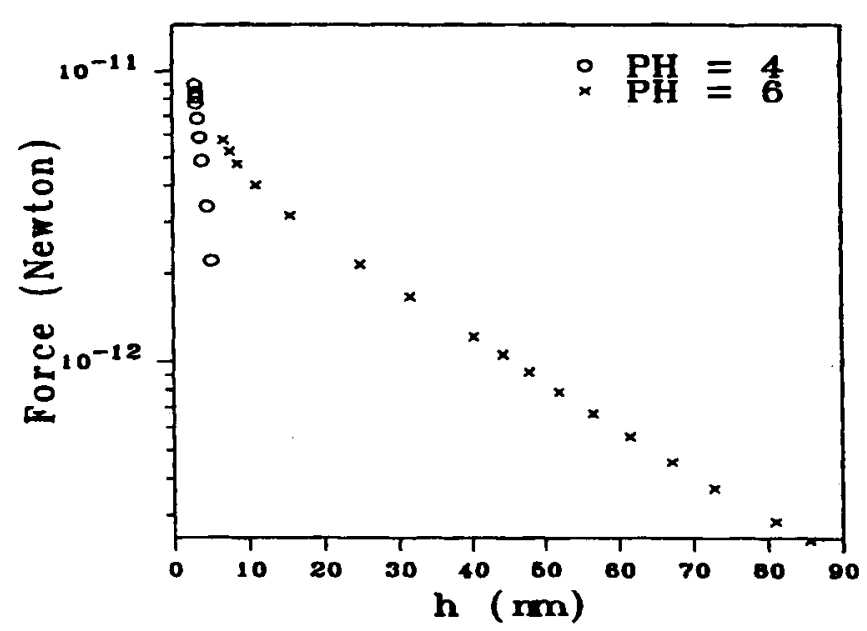

Figure 3. Force profile with NP10 at two different $\mathrm{pH}$ values 6 and 4 . of magnetic field, it can also be easily found out from the stationary Bragg peak. Another way of checking during the force measurement is by stopping the magnetic field and restarting again. If the peak disappears (when field is zero) and reappears (for nonzero field) in the same position as it was earlier for a given field, it is clear that the droplets are re-dispersible and aggregates free. The electrostatic repulsive forces between the droplets $(a=94 \mathrm{~nm})$ and the oxide grains $(a=5 \mathrm{~nm})$ at a spacing of $20 \mathrm{~nm}$ is $2.37 \times 10^{-12} \mathrm{~N}$ and $9.2 \times 10^{-14} \mathrm{~N}$, respectively. So, it is clear that the range repulsive forces between the oxide grains is about two orders of magnitude less compared to the forces between the droplets.

At a $\mathrm{pH}$ of 4 , the estimated value of debye length is $30.4 \mathrm{~nm}$ and the corresponding charge density $(\sigma)$ is

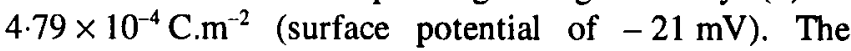
estimated value of repulsive force at this charge density for different inter droplet separations $10 \mathrm{~nm}$ and $80 \mathrm{~nm}$ are $5.20 \times 10^{-12} \mathrm{~N}$ and $3.17 \times 10^{-13} \mathrm{~N}$, respectively. If there is any contribution due to electrostatic repulsion, our force profiles at $\mathrm{pH}=4$, would have followed the above values. On the contrary, we do not see any repulsion till an inter droplet spacing of about $15 \mathrm{~nm}$. So, the contributions due to electrostatic repulsion at this $\mathrm{pH}$ value can be dismissed.

Similarly, for a $\mathrm{pH}=6$, the estimated value of surface charge density $(\sigma)$ is $4.16 \times 10^{-5} \mathrm{C} . \mathrm{m}^{-2}$ (debye length $=250 \mathrm{~nm}$ and surface potential $-15 \mathrm{mV}$ ). The theoretically obtained values of repulsive forces at two inter droplet separations 20 and $80 \mathrm{~nm}$ are respectively $8.54 \times 10^{-13}$ and $3.43 \times 10^{-13} \mathrm{~N}$. However, the experimentally observed value at $80 \mathrm{~nm}$ was the same as that of the theoretically obtained value, whereas at $20 \mathrm{~nm}$, the experimental value was slightly larger than the theoretical value. This observation may imply that at high $\mathrm{pH}$ values, the contribution due to the electrostatic repulsion is significant.

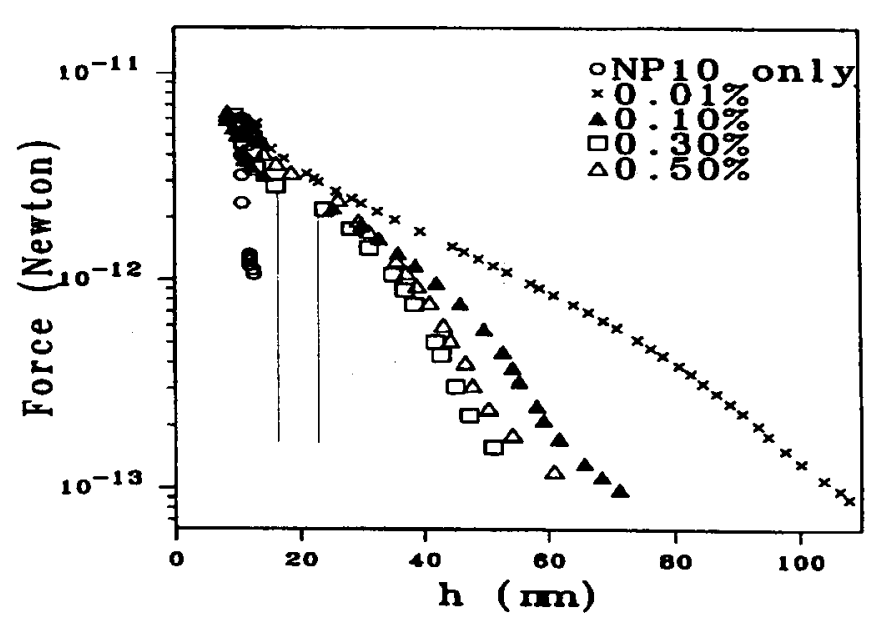

Figure 4. Force-distance profile with PAA $(\mathrm{MW}=250 \mathrm{~K})$ at different concentrations. 
4.3 Force profiles in the presence of PAA at the droplet interface

4.3a Concentration effect: Figure 4 shows the variation of force profiles as a function of distance for PAA solutions of four different concentrations 0.01 and 0.1 , 0.3 and $0.5 \%(w t)$. In all these cases, the pH of PAA solutions was about 4.0. The colloidal droplets are stabilized by a non-ionic surfactant (NP10). Without adsorbed polyelectrolyte, the hard wall profile observed is shown by the circles. Due to the presence of the non-ionic surfactant at the colloidal droplet interface, the system remains perfectly stable even after they are brought down to the hard wall contact.

At low PAA concentration $(0.01 \%)$ the repulsive force profile was long range. Repulsion is first noted at an inter droplet separation of $109 \mathrm{~nm}$. This distance corresponds to more than $3 \mathrm{Rg}$ of PAA. As the distance between the droplets is reduced, an increase in the repulsive force is seen that becomes stronger as the adsorbed PAA begins to overlap. The net repulsion contains contributions due to electrostatic and steric forces. The hard wall contact occurs at a few nanometers further from the surface (i.e. almost close to the one without PAA). This observation suggests that the polyelectrolyte adsorbed at the interface of the droplet is either desorbing from the droplet interface or the tails prefer to flow out from the interdroplet spacing as the separation is reduced. Therefore, at shorter inter droplet spacings, only a few trains remain on the surface of the colloidal droplets. This could be probably because PAA has no specific anchoring groups to get adsorbed irreversibly on the droplets. Due to this weak binding, the layers may be highly dynamic, with individual segments continually attaching and detaching from the droplets.

At low concentrations of PAA $(\mathrm{pH}=4)$, the estimated charge density is about $4.79 \times 10^{-4} \mathrm{C} . \mathrm{m}^{-2}$ and the electrostatic repulsive force value at $100 \mathrm{~nm}$ inter droplet separation is $1.53 \times 10^{-13}$ Newtons. Up to an inter droplet separation of $40 \mathrm{~nm}$, the observed values agree with the theoretically estimated electrostatic force profiles. The surface potential and debye length for this region are $-21 \mathrm{mV}$ and $30.4 \mathrm{~nm}$ respectively. The slope of the force profile is different for the interdroplet spacing of $15 \mathrm{~nm}-40 \mathrm{~nm}$. We compare our results with the steric repulsive force measurement data obtained between a mica surface and polymer solution using surface force apparatus (Israelachvili 1985). For polystyrene with a $M W=140 \mathrm{~K}$, having a flory radius of $32 \mathrm{~nm}$, the experimental results were found to be in good agreement with Alexander-de Gennes model (Israelachvili 1985). In our case the force profiles were not following the above model. This could probably be due to the dominance of electrostatic repulsive contribution over the steric repulsion due to the presence of charges on the polyelectrolyte coils. Whereas with neutral polymers the contribution is purely due to the steric repulsion. The computed and experimental values of the repulsive force $(F / R)$ at a spacing of $110 \mathrm{~nm}$ (between mica surface and polystyrene: $\mathrm{Rg}=32 \mathrm{~nm}, \mathrm{MW}=140 \mathrm{~K}$ ) is reported as $10^{-4} \mathrm{Nm}^{-1}$. In our case, where the $\mathrm{MW}=250 \mathrm{~K}$ and
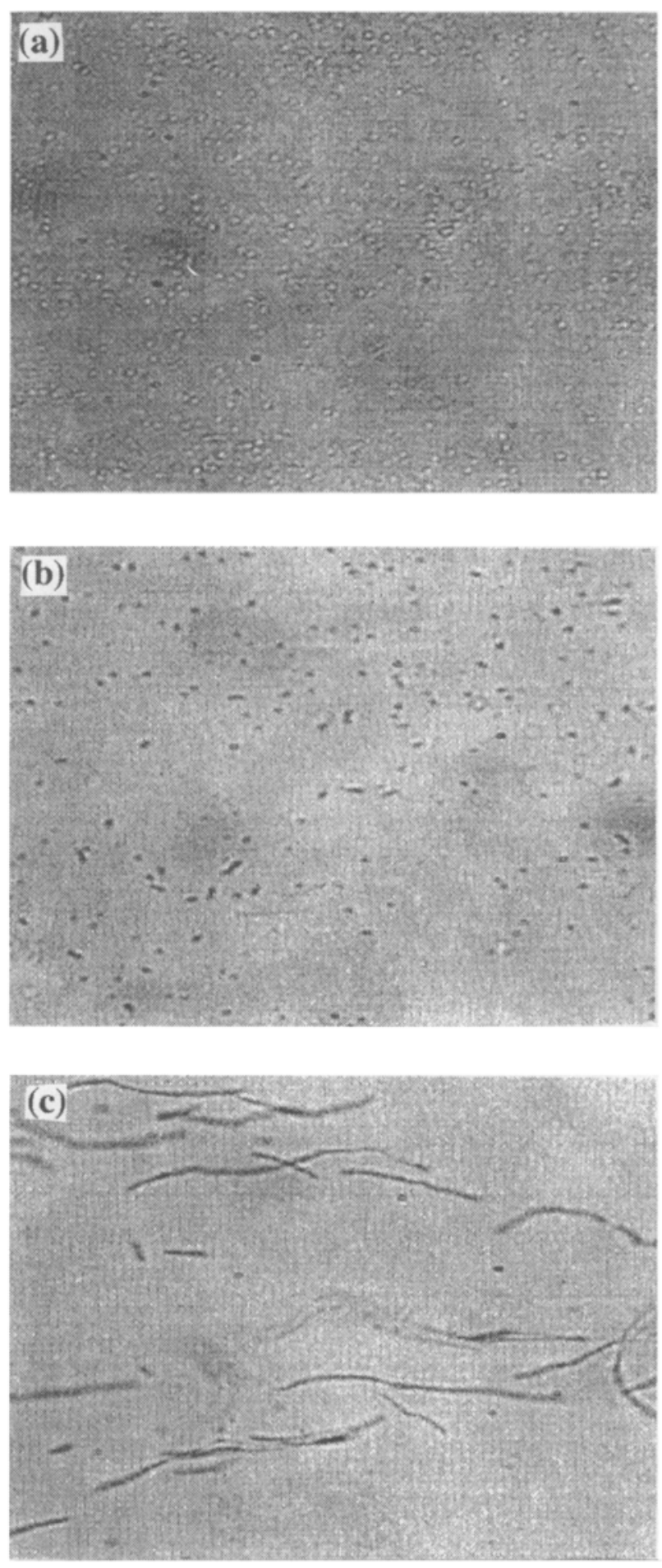

Figure 5. (a) Monodispersed ferrofluid emulsion with $0.1 \%$ PAA solution before subject to magnetic field, (b) irreversible chains (mostly doublets and triplets) observed under the microscope and (c) longer irreversible chains observed under microscope after subjecting it to high magnetic field. 
$\mathrm{Rg}=35 \mathrm{~nm}$, the experimental value of repulsive force (F/R) was about $10^{-6} \mathrm{Nm}^{-1}\left(10^{-13} \mathrm{~N} /\right.$ radius of the droplet $)$. This value is about two orders of magnitude less than the steric repulsive force. This observation further strengthens our argument on the electrostatic force acting at larger distances. Also, the estimated repulsive force at an inter droplet spacing of $100 \mathrm{~nm}$ is found to be $1.53 \times 10^{-13}$ Newtons (charge density $=4.79 \times 10^{-4} \mathrm{C} . \mathrm{m}^{-2}$, surface potential $=-21 \mathrm{mV}$ and debye length $=30.4 \mathrm{~nm}$ ) in good agreement with the observed value.

4.3b Polyelectrolyte bridging: Concentration up to $0.01 \%$ of the colloidal system was perfectly stable and the force curves were reversible. At higher concentrations e.g. $0.1 \%$, when the droplet separation was about $25 \mathrm{~nm}$ from the hard wall touching, we noticed a jump (instability region shown by two vertical lines in figure 4) on the force profile. In figure 4 , we have shown only two PAA concentrations 0.01 and $0.1 \%$, however, experimental results at intermediate concentration were similar to what is observed with $0.1 \%$ PAA concentration. Microscopic observation of the colloidal system just before the instability region shows that doublets or chains are not formed until this level (figure 5a). Just after the jump, we observed formation of doublets and singlets in the system (figure 5b). Further reduction in the separation (with larger magnetic field) between the droplets increases the number of these doublets. It also leads to the formation of longer chains with several droplets attached (figure 5c). Probably, this is due to the 'bridging' of two or more polymer-covered surfaces, leading to permanent chains even after removing the field. Just after the instability region, only a few trains are remaining on each droplet. As the train length varies from one droplet to another, we see the first doublet when the longest train connects two droplets. As we reduce the separation further, the remaining smaller trains start to join and therefore the number of droplets in each chains and the length of the chain increases progressively. This observation is in tune with the argument of Lyklema and Fleer (1987) that the electrostatic repulsion dominates the interaction at longer surface separation and bridge formation occur only at smaller separation. In a recent report (Semenor and Joanny 1995), it has been shown that the adsorbed polymer on the surface can be divided into two regions-an inner layer close to the adsorbing surface essentially by loops and the other layer contains mostly chain tails. The cross over between these two layers occurs at an intermediate distance, much smaller than the radius of gyration $\mathrm{Rg}$. In our case the bridging takes place in this cross over region. However, using MCT we could not directly measure the exact value of this attractive binding force.

The emulsion becomes unstable at $\mathrm{pH}<3$. The bridging phenomena was observed at $\mathrm{pH}=4$ or above where the ferrofluid emulsion was perfectly stable. It should be noted that the 'unstable' condition at $\mathrm{pH}=3$ or lower is entirely different from that of the 'bridging' condition. In the former case, lots of droplets stick together (clusters or aggregates) naturally without any magnetic field and the process is irreversible. Whereas in the latter case, the droplets form one droplet thick long chains when subjected to a magnetic field and the chains broke apart with time (reversible) and the droplets are completely re-dispersed when subjected to ultrasound. This chaining occurs only in the presence of PAA with a concentration above $0.1 \mathrm{wt} \%$.

Figure 5a shows the monodispersed colloidal droplets stabilized with PAA before subjecting to any field. Figure $5 \mathrm{~b}$ shows the irreversible doublets and triplets or short chains formed when the droplets are brought together to a distance close to $20 \mathrm{~nm}$. On further reduction in the droplet separation by increasing the magnetic field leads to larger irreversible chains (figure $5 \mathrm{c}$ ) formation. Earlier, it has been reported that a long range attractive force between two solid surfaces in good polymer solution (Klien and Luckham 1984) and this attraction was found to be dependent on the extent of the polymer adsorbed on the surface. At full coverage, the attraction disappeared and long range repulsion alone is observed. However, the observed attraction was at a distance much larger than the radius of gyration of the polymer used. In our case, we notice long range repulsion even immediately after the addition of PAA in the system and the bridging takes place at a distance much smaller than the radius of gyration of the adsorbed polyelectrolyte.

Microscopic observations also confirm that the doublet and triplet formation occurs at the same distance. In our case, the bridges have been observed only at high PAA concentrations (above $0.1 \%$ ). At $0.01 \%$, no chaining has been noticed and the system was perfectly stable even at very high field. These observations show that an optimum PAA concentration is supposed to prevent an eventual binding between the colloidal droplets but sufficient enough to induce long range forces.

4.3c Time dependence on the adsorption of PAA: Figure 6 shows the force versus distance, for the charged ferrofluid droplets with PAA at the interface $(0.01 \%)$ as a function of time. The force profile is slightly shifted towards the short range region with an increase in the incubation time, without altering the slope of the profile. Even after $48 \mathrm{~h}$ of incubation, no considerable change in the force profile is noticed. A small shift in the force profile towards the shorter range could be due to the fact that the adsorbed polyelectrolyte is adopting a flattened conformation (more number of loops than tails) with time. However, earlier studies (Klien and Luchham 1984) on mica surface showed that the range of repulsion increases with increase in incubation time. This obser- 
vation shows that nature of conformation of polyelectrolyte on the colloidal liquid droplet is different from that of the mica surface.

4.3d Effect of salt: Figure 7 shows the force profile for different salt concentrations at a fixed PAA concentration. In all these cases, the $\mathrm{pH}$ of the solutions were fixed at 4. Let us first consider a situation where there is no PAA in the system. Under this condition, if we simply increase the salt concentration from $0.001 \mathrm{M}$ to $1.0 \mathrm{M}$, the diffused double layer (debye length) is reduced from a value of about $10 \mathrm{~nm}$ to $0.3 \mathrm{~nm}$. This means that the range of repulsion should reduce considerably when the salt concentration increases from $0.001 \mathrm{M}$ to $1.0 \mathrm{M}$. But what we see in figure 7 , is different from what is expected according to the concept of electrostatic screening theory. Without any salt in the system, for a PAA concentration of $0.01 \%$, the force profile is a hard sphere profile (shown by the circles). As the salt concentration increases from $0.001 \mathrm{M}$ to $0.1 \mathrm{M} \mathrm{NaCl}$, the range of repulsion increases. On further increase in the salt concentration the range of repulsion reduces.

The hydrodynamic diameter of the droplet with and without PAA at the interface was measured from dynamic light scattering (DLS) using an Amtec Spectrometer in association with a Brook Heaven Instrument Digital correlator (BI 2030AT, 72 Channel correlator). Figure 8 shows the hydrodynamic diameter of the droplet which is about $200 \mathrm{~nm}$ for a salt concentration of $0.001 \mathrm{M}$. This means an increase of about $10 \mathrm{~nm}$ from the bare droplet, without any salt. At $0.1 \mathrm{M} \mathrm{NaCl}$, the hydrodynamic radius is increased to $400 \mathrm{~nm}$. Further increase in the salt concentration leads to a reduction in the hydrodynamic thickness to $300 \mathrm{~nm}$. These results suggest that the addition of salt in the system has a dramatic influence on the adsorption isotherm. However, this result is not sufficient enough to obtain the information about

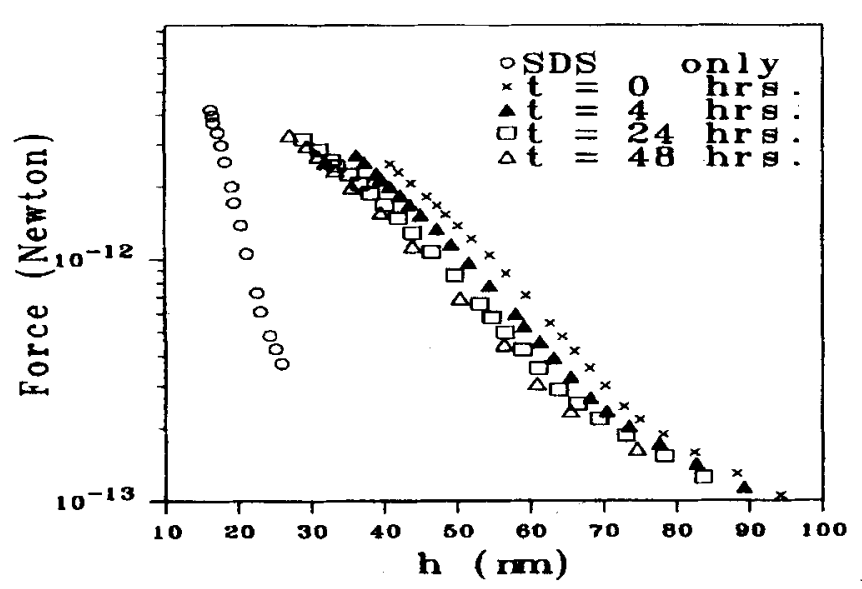

Figure 6. Force profile with PAA $(250 \mathrm{~K}, 0.1 \% \mathrm{MW})$ at various incubation time. the possible conformation of the polyelectrolytes on the droplet surface.

A recent study (Klien and Luckham 1984) on the adsorption excess of polyelectrolyte (gelatin) on mica surface shows a monotonic increase in the adsorption excess, with an increase in the salt concentration. The above effect is attributed to the electrostatic binding between the positively charged groups on the gelatin with the negatively charged mica surface and the short range van der Waal or hydrophobic interaction between the dissociated groups of polyelectrolytes and the mica surface. However, the force measurements on the same system show a reduction in the range of repulsion due to screening at high salt concentrations. Moreover, all their force profiles (at different salt concentrations and $\mathrm{pH})$ are found to follow the Alexander-de Gennes model. But in our case, the adsorption characteristics are similar to that of the gelatin but on the force profile we do not see any screening effect with an increase in the salt concentration up to $0.1 \mathrm{M} \mathrm{NaCl}$.

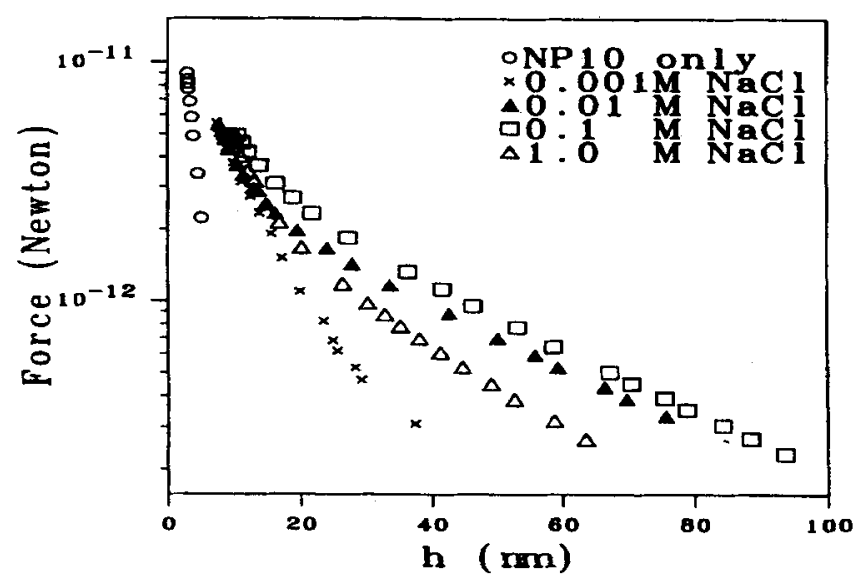

Figure 7. Force profile with PAA $(250 \mathrm{~K}, 0.01 \% \mathrm{MW})$ for various $\mathrm{NaCl}$ concentrations.

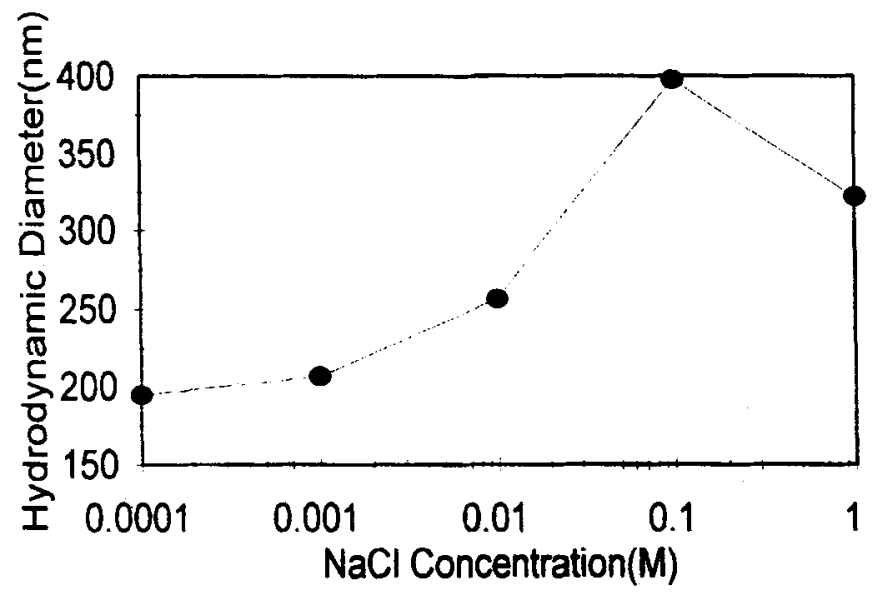

Figure 8. Hydrodynamic radius of the droplets for different salt concentrations. 
According to the extended self-consistent field theory of Scheutjeens and Fleer $(1979,1980)$, for weak polyelectrolytes, the length of the tails increases rapidly with an increase in the salt concentration while the trains decrease slightly. Increase in the adsorption on the surface with the increase in salt is considered due to the penetration of salt ions between the polyelectrolyte segment that in turn reduces the segment-segment repulsion. Earlier studies (Papenhuijzen et al 1985) on the adsorption of polystyrene sulphonate on polyoxymethylene crystals show a monotonic increase in the adsorption excess with increasing salt concentration up to $3 \mathrm{M} \mathrm{NaCl}$. Adsorption of poly(styrene sulphonate) (Cosgrove et al 1986) and polysilanes (Lyklema 1985) on polystyrene latex particles also show a similar strong dependence on the ionic strength. Therefore, the observed increase in the hydrodynamic thickness of the ferrofluid droplet with salt concentration is not surprising in terms of the earlier results on solid surfaces.

\section{Conclusions}

We have studied the repulsive forces in the presence of polyelectrolytes adsorbed at the interface of colloidal droplets using magnetic chaining technique. We have observed a long range repulsive force profiles up to a PAA concentration of $0.01 \%$. Above $0.01 \%$ of PAA, and at short interdroplet distance, irreversible chaining is observed due to polyelectrolyte bridging. Incubation time for polymer adsorption on the ferrofluid interface has found to have no influence on the force profiles. The range of repulsion and the hydrodynamic radius of the droplets is found to be increasing with salt concentration up to $0 \cdot 1 \mathrm{M} \mathrm{NaCl}$.

\section{Acknowledgements}

One of the authors (JP) would like to thank $\mathrm{Dr} T$ Jayakumar, Shri P Kalyanasundaram, Dr Baldev Raj and Dr Placid Rodriguez, Indira Gandhi Centre for Atomic Research, Kalpakkam, for constant encouragement and support. Authors thank Rhone Poulenc Inc (Paris) for financial assistance.

\section{References}

Becher P 1965 Emulsions: theory and practice (New York: Rheinhold)

Bibette J 1991 J. Colloid Interface Sci. 147474
Blakmeer J, Bohmer M R, Cohen Stuart M A and Fleer G J 1990 Macromolecules 232301

Bohmer M R, Evers O A and Scheutjens J M H M 1990 Macromolecules 232288

Brandrupp J and Immergutt E G 1975 Polymer hand book (New York: Wiley Interscience) 2nd ed

Cosgrove T, Obey T M and Vincent B $1986 \mathrm{~J}$. Coll. Int. Sci. 11409

Derjaguin B 1940 Trans. Faraday Soc. 36203

Hansma D K, Elings V B, Marto J and Bracker C E 1988 Langmuir 242209

Israelachvili J N 1985 Intermolecular and surface forces (San Diego: Academic Press)

Israelachvili J N and Tabor D 1972 Proc. R. Soc. London Ser. A. 33119

Israelachvili J N and Adams G E $1978 J C S$ Faraday Trans. I 74975

Klien J and Luckham P 1984 Nature 308836

Leal-Calderon F, Stora T, Mondain-Monval $O$ and Bibette $J$ 1994 Phys. Rev. Letts. 72865

Luckham P F and Klien J 1984 J. Chem. Soc. Faraday Trans. I 80865

Lyklema J 1985 Modern trends of colloid science (ed.) H F Eicke (Basel: Birkhauser Verlag)

Lyklema J and Fleer G 1987 J. Colloids Surf. 25357

Mondain-Monval O, Leal Calderon F, Philip J and Bibette J 1995 Phys. Rev. Lett. 753364

Mou T, Flores G A, Liu J, Bibette J and Richard J 1994 lnt. J. Mod. Phys. B8 2779

Napper D H 1983 Polymeric stabilization of colloidal dispersions (New York: Academic Press)

Papenhuijzen J, Fleer G J and Bijsterbisch B H $1985 \mathrm{~J}$. Coll. Inter. Sci. 104530,553

Patel S and Tirrell M 1989 Ann. Rev. Phy. Chem. 40597

Philip J 1995 Rhone Poulenc Research Report, Paris

Philip J, Monval O M, Calderon L F and Bibette J 1997 J. Phys. D. Appl. Phys. 302798

Prieve D C and Frej N A 1990 Langmuir 6396

Rosensweig R E 1985 Ferrohydrodynamics (New York: Cambridge University Press)

Sato T and Ruchs R 1980 Stabilization of colloidal dispersions by polymer adsorption (New York: Dekker)

Scheutjens J M H M and Fleer G J 1979 J. Phys. Chem. 83 163

Scheutjens J M H M and Fleer G J 1980 J. Phys. Chem 84178

Semenor A N and Joanny J F 1995 Euro. Phy. Lett. 29279

Th Tadors 1983 Solid state dispersions (New York: Academic Press)

Th Tadors 1988 Polymers in colloidal systems (Amsterdam: Elsevier)

Van der Schee H A and Lyklema J 1984 J. Phys. Chem. 88 6661

Verwey E J W and Overbeck J T H 1948 Theory of the stability of lyophophic colloids (Amsterdam: Elsevier) 